\title{
Gliomatosis peritonei: a clinicopathologic and immunohistochemical study of 21 cases
}

\author{
Li Liang ${ }^{1}$, Yifen Zhang ${ }^{1,2}$, Anais Malpica ${ }^{1}$, Preetha Ramalingam ${ }^{1}$, Elizabeth D Euscher ${ }^{1}$, \\ Gregory N Fuller ${ }^{1}$ and Jinsong Liu ${ }^{1,3}$ \\ ${ }^{1}$ Department of Pathology, The University of Texas MD Anderson Cancer Center, Houston, TX, USA; \\ ${ }^{2}$ Department of Pathology, Nanjing Drum Tower Hospital, Nanjing University Medical School, Nanjing, \\ People's Republic of China and ${ }^{3}$ Department of Pathology, The First Affiliated Hospital of Nanjing Medical \\ University, Nanjing, People's Republic of China
}

\begin{abstract}
Gliomatosis peritonei, a rare condition often associated with immature ovarian teratoma, is characterized by the presence of mature glial tissue in the peritoneum. We retrospectively evaluated 21 patients with gliomatosis peritonei and studied their clinicopathologic features and immunophenotype. The patients' ages ranged from 5 to 42 years (median, 19 years). Their primary ovarian tumors consisted of immature teratoma $(n=14)$, mixed germ cell tumors $(n=6)$, and mature teratoma with a carcinoid tumor $(n=1)$. Gliomatosis peritonei was diagnosed at the same time as primary ovarian neoplasm in 16 patients and secondary surgery in 5 patients. Also, 11 of 21 patients had metastatic immature teratoma $(n=4)$, metastatic mature teratoma $(n=2)$, or both $(n=5)$. One patient developed glioma arising from gliomatosis peritonei. Seventeen patients had follow-up information and were alive with no evidence of disease $(n=13)$, alive with disease $(n=3)$, or alive with an unknown disease status $(n=1)$. The follow-up durations ranged from 1 to 229 months (mean, 49 months; median, 23 months). Immunohistochemistry results demonstrated that SOX2 was expressed in all cases of gliomatosis peritonei and glioma with tissue available (nine of nine cases), whereas OCT4 and NANOG were negative in all cases with available tissue (eight of eight cases). In conclusion, both gliomatosis peritonei and glioma arising from it show a SOX2+/OCT4-/NANOG - immunophenotype. These findings demonstrated that gliomatosis peritonei is associated with favorable prognosis, although it is important to rule out potentially associated immature teratoma and malignant transformation. SOX2 may have an important role in the development of gliomatosis peritonei.
\end{abstract}

Modern Pathology (2015) 28, 1613-1620; doi:10.1038/modpathol.2015.116; published online 13 November 2015

Gliomatosis peritonei, which is characterized by mature glial tissue in the peritoneum, is a rare condition usually associated with immature ovarian teratoma ${ }^{1-5}$ and in rare cases with ventriculoperitoneal shunting. ${ }^{6-8}$ Pathologically, gliomatosis peritonei is considered to be grade 0 teratoma according to the World Health Organization grading system used for immature teratoma. ${ }^{9}$ The presence of gliomatosis peritonei, regardless of its extent, is usually not associated with adverse outcomes, ${ }^{10}$ however, gliomatosis peritonei has been reported to transform into malignant glial neoplasms. ${ }^{11,12}$ The origin of gliomatosis peritonei is also poorly understood.

Correspondence: Dr J Liu, MD, PhD, Department of Pathology, The University of Texas MD Anderson Cancer Center, Unit 85, 1515 Holcombe Boulevard, Houston, TX 77030, USA.

E-mail: jliu@mdanderson.org

Received 27 May 2015; revised 27 August 2015; accepted 29 August 2015; published online 13 November 2015
In the present study, we examined the clinicopathologic features of gliomatosis peritonei in 21 patients and the expression of a panel of stem cell markers consisting of POU5F1 (POU domain, class 5, transcription factor 1), also known as octamerbinding transcription factor 4 (OCT4), homeobox protein NANOG, and sex determining region Y-box 2 (SOX2).

\section{Materials and methods}

Tissue Specimens

A search of the pathology files at the University of Texas, MD Anderson Cancer Center from 1988 to 2014 performed after Institutional Review Board approval of the study identified 21 cases of gliomatosis peritonei. Relevant clinical data on the patients were obtained via retrospective review of the patients' medical files. These data included 
demographic information, diagnosis, and tumor grade. Their follow-up information was updated through June 2015 via review of their medical records and tumor registration. Hematoxylin and eosin (H\&E)stained slides containing tissue sections of gliomatosis peritonei were reviewed. Paraffin-embedded tissue blocks $(n=8)$ and an unstained slide containing gliomatosis peritonei and/or glioma were used for immunohistochemical staining for SOX2, OCT4, and NANOG. The patients' histopathologic diagnoses were based on the fourth edition of the World Health Organization Classification of Tumours of Female Reproductive Organs. ${ }^{9}$ Immature teratomas were graded using a two-tiered system (low and high grade). ${ }^{13}$ The tumors were staged using the International Federation of Gynecology and Obstetrics 2013 Ovarian Cancer Staging System. ${ }^{14}$

\section{Immunohistochemical Staining}

Immunohistochemical staining for SOX2 using the rabbit monoclonal antibody D6D9 (1:100 dilution; Cell Signaling Technology, Danvers, MA, USA), OCT4 using a rabbit polyclonal antibody (1:200 dilution; Cell Signaling Technology), and NANOG using the rabbit monoclonal antibody D73G4 (1:800 dilution; Cell Signaling Technology) was performed. After tumor sections were deparaffinized, rehydrated, and blocked using Peroxidazed 1 endogenous peroxidase blocker (PX968; Biocare Medical, Concord, CA, USA) at room temperature for $5 \mathrm{~min}$, antigen retrieval was performed using Universal Decloaker buffer solution (UD1000M; Biocare Medical) in an autoclave at $125^{\circ} \mathrm{C}$ for $30 \mathrm{~s}$ and $90{ }^{\circ} \mathrm{C}$ for $10 \mathrm{~s}$. Nonspecific binding was blocked with Background Sniper blocking reagent (BS966M; Biocare Medical) for $30 \mathrm{~min}$ at room temperature. Slides were incubated with the primary antibodies overnight at $4^{\circ} \mathrm{C}$, a biotin-labeled secondary antibody (Universal Goat Link (GU600H); Biocare Medical) for $10 \mathrm{~min}$, and 4plus HRP 1000 Universal Detection (HP604; Biocare Medical) for $10 \mathrm{~min}$. After being stained with 3,3'-diaminobenzidine chromogen (DB801L; Biocare Medical), the sections were counterstained with hematoxylin, dehydrated, and mounted. Only nuclear staining for OCT4, NANOG, and SOX2 was considered to be positive.

\section{Results}

\section{Clinical and Follow-Up Information}

At the time of diagnosis of gliomatosis peritonei, the patients ranged in age from 5 to 42 years (mean, 22 years; median, 19 years). The clinicopathologic features of all cases were summarized in Table 1 and Table 2. Eight of the patients were white, eight were Hispanic, two were black, and one was Asian; information on race was not available for two patients. A total of 9 of $21(43 \%)$ cases were consultation cases submitted by outside institutions. Seventeen patients had follow-up information and were alive with no evidence of disease $(n=13)$, alive with disease according to restaging computed tomography (CT) scan $(n=3)$, or alive with an unknown disease status $(n=1)$. Their follow-up durations ranged from 1 to 229 months (mean, 49 months; median, 23 months). Four patients received chemotherapy before the diagnosis of gliomatosis peritonei (Table 2). Two of these four patients also developed growing teratoma syndrome (patient numbers 10 and 12), which was confirmed by CT scan (Table 2). One patient developed glioma arising from gliomatosis peritonei. She first presented with abdominal distention, weight loss, and ascites. Both CT scan and magnetic resonance imaging (MRI) showed a 29.0-cm pelvic mass. She underwent unilateral salpingo-oophorectomy, partial omentectomy, and was diagnosed with high-grade immature teratoma. Subsequently, patient received four cycles of etoposide and cisplatin chemotherapy. Follow-up CT and MRI showed sheet-like nodularity along the peritoneum and omental caking. She underwent cytoreductive surgery and hyperthermic intraperitoneal chemotherapy with cisplatin. A diagnosis of glioma arising from gliomatosis peritonei was made at that point. Patient received postoperative radiotherapy. She was alive with disease 4 months after the diagnosis of glioma (10 months after the diagnosis of ovarian immature teratoma).

\section{Histopathology and Immunohistochemical Profiles}

The primary ovarian neoplasms in this study were immature teratomas $(n=14)$, mixed germ cell tumors with immature teratoma components $(n=6)$, and a mature teratoma with a carcinoid tumor $(n=1)$. Of the 14 immature teratomas, 5 were low grade, and 9 were high grade (Table 1). Eleven of 21 (52\%) patients had metastatic immature teratoma $(n=4)$, metastatic mature teratoma $(n=2)$, or both $(n=5)$. One patients had mature teratoma associated with a carcinoid tumor (case number 14).

Gliomatosis peritonei was diagnosed at the original surgery in 15 of 21 (71\%) patients and at secondary surgery in 6 of 21 (29\%) patients (Table 2). In addition, gliomatosis peritonei was diagnosed using H\&E-stained slides in 15 of 21 (71\%) cases, which were characterized by mature glial tissue in the peritoneum (Figure 1a and b). Gliomatosis peritonei formed individual nodules on the surface of the peritoneum or a mass-like lesion. Immunohistochemical staining for glial fibrillary acidic protein (GFAP) was performed in five cases, which demonstrated positive cytoplasmic staining, supporting the diagnosis of gliomatosis peritonei. Other immunohistochemical staining performed included that for S100 (positive in two cases), SALL4 (negative in one case), pan-cytokeratin (negative in one case), and CD99 (negative in one case). 
Table 1 Clinicopathologic features of the 21 cases of gliomatosis peritonei

\begin{tabular}{|c|c|c|c|c|c|c|c|c|c|c|c|}
\hline$P T$ & $\begin{array}{l}\text { Age, } \\
\text { years }\end{array}$ & Ovarian neoplasm & $\begin{array}{l}\text { FIGO } \\
\text { stage }^{\mathrm{a}}\end{array}$ & $\begin{array}{l}\text { Metastatic immature } \\
\text { teratoma }^{\mathrm{b}}\end{array}$ & $\begin{array}{l}\text { Metastatic mature } \\
\text { teratoma }^{\mathrm{b}}\end{array}$ & $\begin{array}{l}\text { Lymph node } \\
\text { status }\end{array}$ & GFAP & SOX2 & OCT4 & NANOG & $\begin{array}{l}\text { Follow-up } \\
\text { duration }^{\mathrm{c}}\end{array}$ \\
\hline 1 & 18 & Immature teratoma, low grade & IC2 & No & No & Nodal gliomatosis & + & NA & NA & NA & ANED, 19 months \\
\hline 2 & 29 & Immature teratoma, low grade & NA & No & No & NA & + & NA & NA & NA & ANED, 2 months \\
\hline 3 & 14 & Immature teratoma, high grade & NA & Yes & No & NA & + & + & - & - & ANED, 92 months \\
\hline 4 & 15 & Immature teratoma, low grade & NA & No & Yes & Mature teratoma & NA & + & NA & NA & NA \\
\hline 5 & 19 & Immature teratoma, high grade & IA & No & Yes & Negative & NA & + & - & - & ANED, 58 months \\
\hline $6^{d}$ & 13 & Mixed germ cell tumor & IA & No & No & Negative & NA & + & - & - & ANED, 71 month \\
\hline $7^{d}$ & 36 & Mixed germ cell tumor & NA & Yes & Yes & NA & NA & NA & NA & NA & ADSU, 1 month \\
\hline 8 & 15 & Immature teratoma, high grade & IA & No & No & NA & NA & + & - & - & ANED, 37 months \\
\hline 9 & 33 & Immature teratoma, low grade & IA & No & No & NA & NA & NA & NA & NA & ANED, 184 months \\
\hline $10^{\mathrm{d}}$ & 19 & Mixed germ cell tumor & IIIC & Yes & Yes & Negative & NA & + & - & - & ANED, 46 months \\
\hline 11 & 22 & Immature teratoma, high grade & IA & No & No & Negative & + & NA & NA & NA & ANED, 15 months \\
\hline $12^{\mathrm{d}, \mathrm{e}}$ & 40 & Mixed germ cell tumor & IIIC & Yes & No & NA & NA & + & - & - & AWD, 29 months \\
\hline $13^{\mathrm{d}, \mathrm{e}}$ & 42 & Mixed germ cell tumor & IIIC & Yes & Yes & Nodal gliomatosis & NA & + & - & - & AWD, 23 months \\
\hline 14 & 5 & $\begin{array}{l}\text { Mature cystic teratoma with } \\
\text { carcinoid tumor }\end{array}$ & NA & No & No & NA & + & NA & NA & NA & ANED, 4 months \\
\hline $15^{\mathrm{e}, \mathrm{f}}$ & 10 & Immature teratoma, high grade & NA & Yes & Yes & NA & NA & + & - & - & AWD, 10 months \\
\hline $16^{\mathrm{d}}$ & 10 & Mixed germ cell tumor & NA & No & No & Nodal gliomatosis & NA & NA & NA & NA & ANED, 11 months \\
\hline 17 & 21 & Immature teratoma, high grade & NA & No & No & NA & NA & NA & NA & NA & NA \\
\hline 18 & 21 & Immature teratoma, high grade & NA & Yes & No & Negative & NA & NA & NA & NA & NA \\
\hline 19 & 14 & Immature teratoma, low grade & NA & No & No & NA & NA & NA & NA & NA & NA \\
\hline 20 & 22 & Immature teratoma, high grade & NA & Yes & Yes & NA & NA & NA & NA & NA & ANED, 5 months \\
\hline 21 & 41 & Immature teratoma, high grade & NA & Yes & No & IT & NA & NA & NA & NA & ANED, 229 months \\
\hline
\end{tabular}

Abbreviations: ADSU, alive, disease status unknown; ANED, alive with no evidence of disease; AWD, alive with disease; FIGO, International Federation of Gynecology and Obstetrics; GFAP, glia fibrillary acidic protein; NA, not available; PT, patient.

${ }^{\mathrm{a}}$ International Federation of Gynecology and Obstetrics 2013 Ovarian Cancer Staging System.

IIdentified at a different anatomic site from that of gliomatosis peritonei.

${ }^{\mathrm{C}}$ Duration of follow-up after the diagnosis of primary ovarian neoplasms. Updated on June 2015.

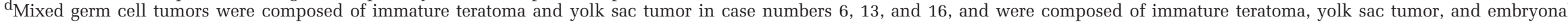
carcinoma in case numbers 7,10 , and 12 .

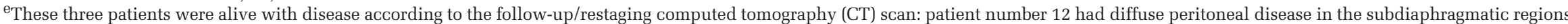

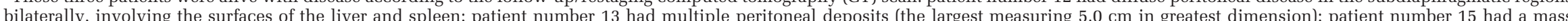
within the cul-de-sac $(\sim 6.0 \mathrm{~cm})$

fatient developed glioma arising from gliomatosis peritonei. 


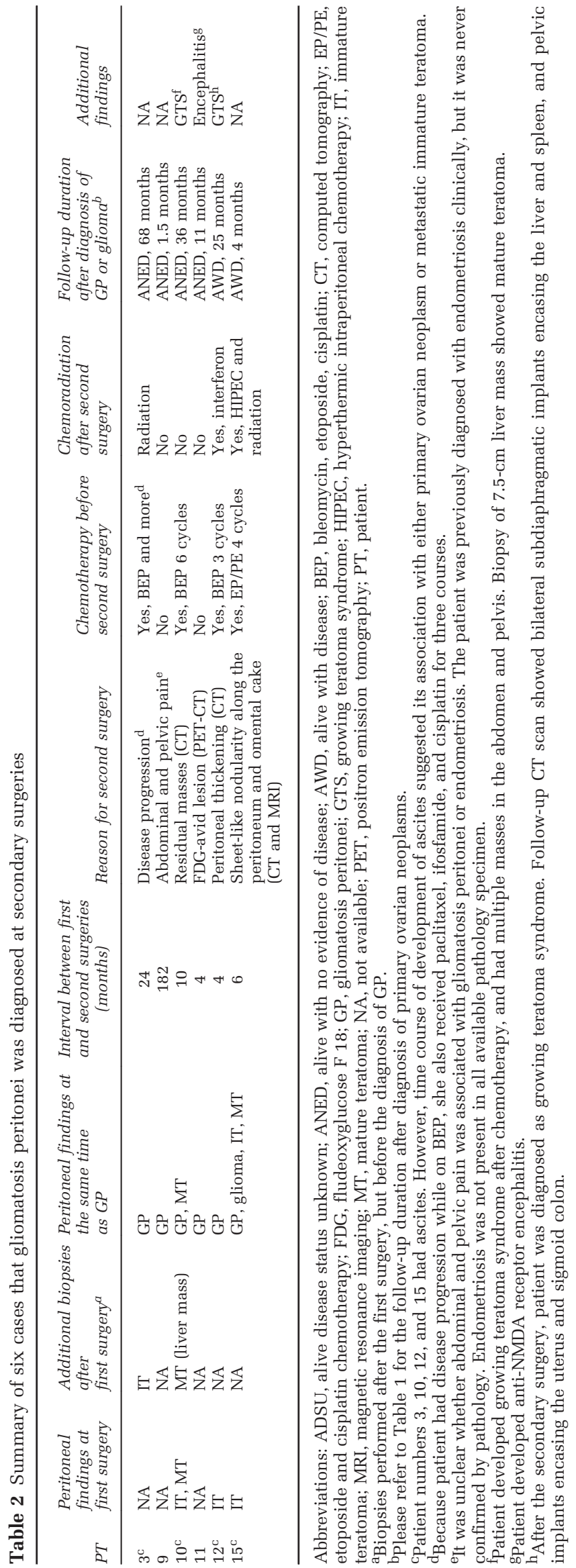

To determine the stem cell origin of gliomatosis peritonei, we performed immunohistochemical staining for OCT4, NANOG, and SOX2 in sections containing gliomatosis peritonei. The results demonstrated that SOX2 (Figure 1c and d) was diffusely expressed in nine of nine cases $(100 \%)$ with either available tissue blocks or unstained slides, whereas OCT4 (Figure 1e) and NANOG (Figure 1f) were not expressed in eight of eight cases with tissue blocks.

One patient developed glioma arising in the setting of gliomatosis peritonei. Tissue sections demonstrated extensive gliomatosis with foci of increased cellularity and nuclear atypia (Figure 2a), abundant mitotic figures with a manual mitotic count revealed 5-7 mitotic figures per 10 high-power fields (Figure 2b), vascular proliferation (Figure 2c), and necrosis (Figure 2d). Quantitative assessment of immunohistochemical staining for Ki-67 (Figure 2e) showed average proliferation index of $22.2 \%$ and quantitative assessment of immunohistochemical staining for phosphohistone $\mathrm{H} 3$ revealed 2.3 mitotic figures per 1000 total nuclei, supporting a diagnosis of high-grade glioma, similar to glioblastoma multiforme (grade IV) from central nervous system. Immunohistochemical staining of specimens of glioma demonstrated that the tumor cells were positive for SOX2 (Figure 2f) but negative for OCT4 and NANOG. Moreover, next-generation sequencing-based analysis of this case of glioma failed to identify any mutations in the coding sequences of 50 genes, including BRAF, PIK3CA, PTEN, IDH1, and IDH2.

\section{Discussion}

In this study, we examined the clinicopathologic and immunohistochemical features of gliomatosis peritonei in one of the largest reported series of such patients in the literature. Gliomatosis peritonei was diagnosed at the same time as primary ovarian neoplasms in the majority of the patients $(71 \%)$. Approximately half of the patients also had metastatic immature teratoma, metastatic mature teratoma, or both. Although gliomatosis peritonei was often diagnosed on H\&E-stained tissue sections, inflammatory cell infiltration, hemorrhage, and choroid plexus can be seen in patients with gliomatosis peritonei, which makes it a diagnostic challenge. Differentiating choroid plexus from low-grade epithelial ovarian tumors may be difficult, especially on intraoperative frozen sections. In these challenging situations, immunohistochemical staining for GFAP may be helpful.

Clinically, gliomatosis peritonei is considered grade 0 teratoma and is usually associated with favorable prognosis and managed conservatively, which is supported by our present findings. However, several conditions must be considered before such a diagnosis is made: First, one must consider the coexistence of metastatic immature and/or 
mature teratoma before making such a diagnosis. Therefore, carefully examining all specimens is important, as the presence of metastatic immature teratoma affects prognosis and alters the treatment course. Second, on rare occasions, malignant transformation of glioma should be also considered as
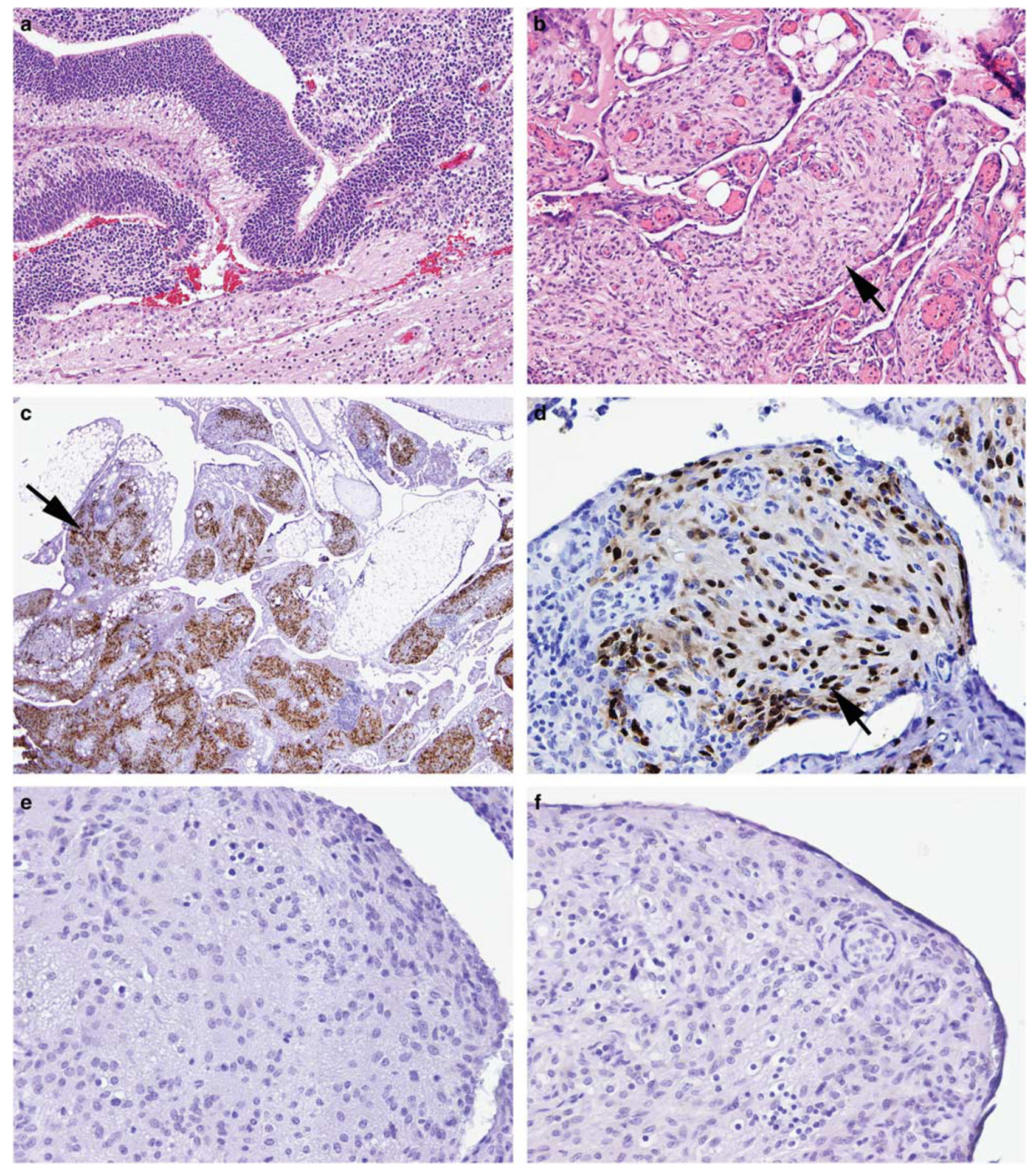

Figure 1 Staining of gliomatosis peritonei specimens for OCT4, NANOG, and SOX2. (a) Patient's ovarian mass was a mixed germ cell tumor, predominantly composed of immature teratoma (hematoxylin and eosin stain; original magnification, $\times 100$ ). (b) Mature glial tissue in the peritoneal cavity demonstrated a micronodular growth pattern, consistent with gliomatosis peritonei (hematoxylin and eosin stain; original magnification, $\times 100$ ). (c and d) Glial cells demonstrated strong, diffuse nuclear staining for SOX2 (immunohistochemical stain; original magnification, $\times 20$ and $\times 200$ in $\mathbf{c}$ and d, respectively). (e) Glial cells were negative for OCT4 (immunohistochemical stain; original magnification, $\times 200$ ). (f) Glial cells were negative for NANOG (immunohistochemical stain; original magnification, $\times 200$ ). 
described here and by other authors. ${ }^{11,12}$ Third, gliomatosis peritonei can be part of growing teratoma syndrome, characterized by increasing growth of metastatic mass that is composed of mature teratoma especially in patients who have received chemotherapy for malignant germ cell tumor. ${ }^{15-20}$
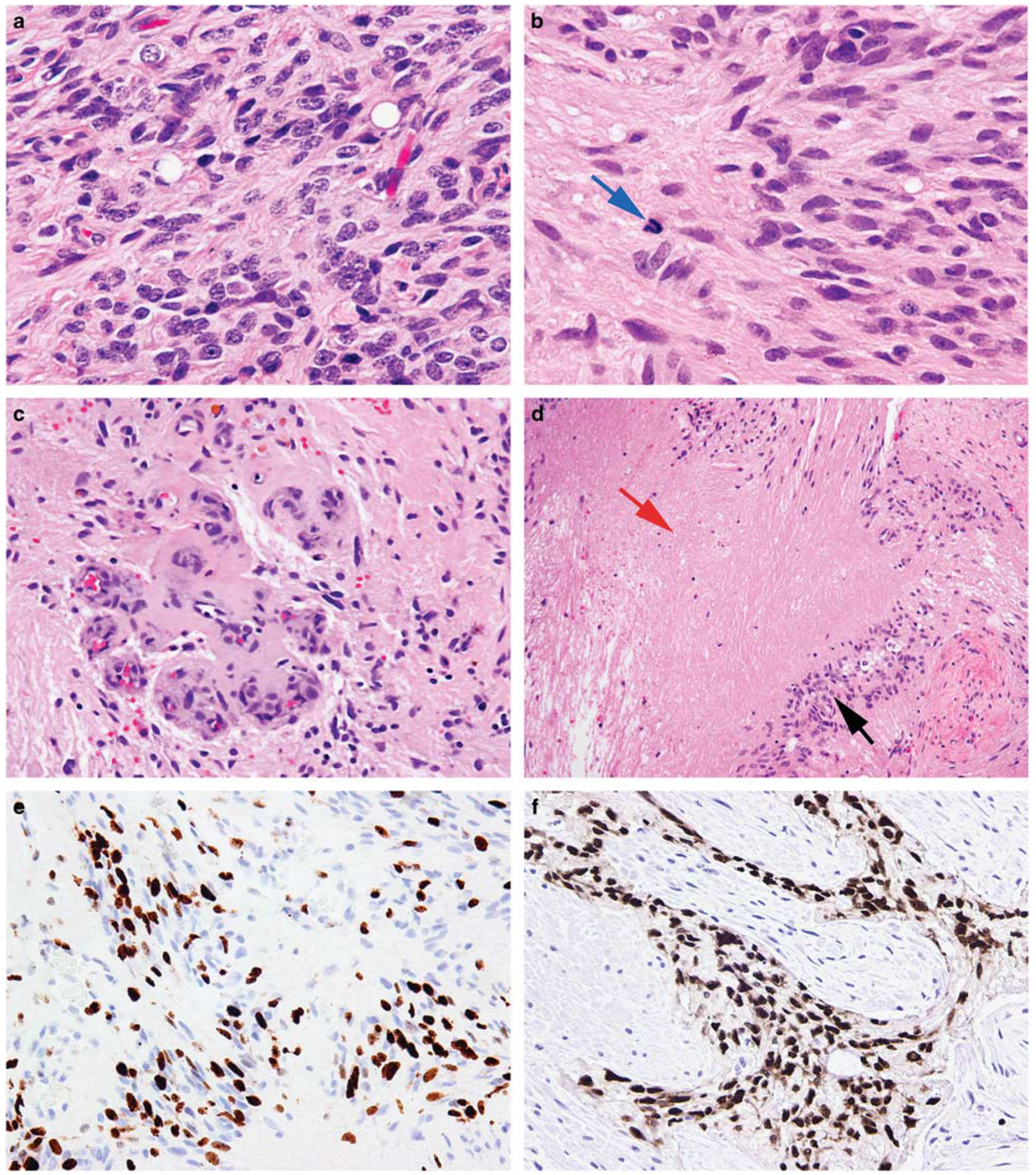

Figure 2 Staining of a specimen of glioma arising from gliomatosis peritonei. (a and $\mathbf{b}$ ) Glioma cells showed increased nuclear atypia and mitotic figures (blue arrow; hematoxylin and eosin stain; original magnification, $\times 400$ ). (c) Vascular proliferation (hematoxylin and eosin stain; original magnification, $\times 200$ ). (d) Glioma cells (black arrow) and necrosis (red arrow; hematoxylin and eosin stain; original magnification, $\times 100)$. (e) Ki-67 immunohistochemical staining demonstrated high proliferative index of glioma (immunohistochemical stain; original magnification, $\times 200$ ). (f) Glioma cells demonstrated positive nuclear staining for SOX2 (immunohistochemical stain; original magnification, $\times 200)$. 


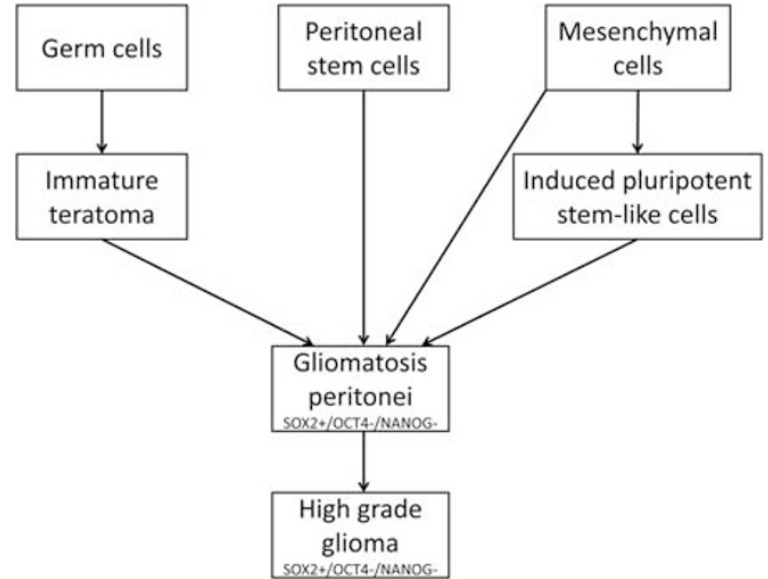

Figure 3 Hypotheses regarding the origin of gliomatosis peritonei. First, it may derive from an immature teratoma that undergoes maturation or from cancer stem cells within an immature teratoma. Second, it may derive from peritoneal stem cells that differentiate toward the neural lineage induced by factors secreted by teratoma. Third, it may derive from subperitoneal mesenchymal cells that transdifferentiate into glial cells either directly or through an intermediate stage of induced pluripotent stemlike cells.

Our results demonstrated that the tumor cells in gliomatosis peritonei specimens expressed SOX2 but not OCT4 or NANOG in all cases with tissue blocks or unstained slides available for immunohistochemical staining, which provides an important molecular clue on the origin of this disease. This is in keeping with the findings reported by Nogales et al. ${ }^{21}$ SOX2 is one of the key factors for maintenance of pluripotency in stem cells ${ }^{22,23}$ and its expression is required for inducing stem cells to differentiate toward the neural lineage. ${ }^{24,25}$ SOX2 is expressed in neural stem cells, ${ }^{26}$ in the majority of glial tumors, ${ }^{27}$ in immature teratoma cases, ${ }^{26}$ and in epithelium of endodermal origin in mature teratoma cases. ${ }^{26}$ Several hypotheses regarding the origin of gliomatosis peritonei have been proposed in the literature (Figure 3), including germ cell origin, as it is frequently associated with germ cell tumors, peritoneal stem cells that differentiate into glial cells under the stimulation of factors secreted by teratomas ${ }^{21,28}$ or transdifferentiation from subperitoneal mesenchymal cells or via induced pluripotent stem-like cells into glial cells. ${ }^{29,30}$ Taken together, our data together with other investigators' finding support a key role for SOX2 in the pathogenesis of gliomatosis peritonei, although detailed mechanisms of how SOX2 induces gliomatosis via one or combinations of three mechanisms mentioned above remains to be determined.

\section{Acknowledgments}

We thank Don Norwood from the Department of Scientific Publications at the University of Texas MD Anderson Cancer Center for editing the manuscript.
This work was supported in part by the grant from the Cancer Prevention and Research Institute of Texas, the MD Anderson Cancer Center SPORE in Ovarian Cancer (National Institutes of Health grant), a Sister Institution Grant from MD Anderson Cancer Center Global Academic Programs. Dr Li Liang was supported by the training grant T32CA163185 from NIH/NCI.

\section{Disclosure/conflict of interest}

The authors declare no conflict of interest.

\section{References}

1 Thurlbeck WM, Scully RE. Solid teratoma of the ovary: a clinocopatholgical analysis of 9 cases. Cancer 1960;13:804-811.

2 Fortt RW, Mathie IK. Gliomatosis peritonei caused by ovarian teratoma. J Clin Pathol 1969;22:348-353.

3 Norris HJ, Zirkin HJ, Benson WL. Immature (malignant) teratoma of the ovary: a clinical and pathologic study of 58 cases. Cancer 1976;37:2359-2372.

4 Robboy SJ, Scully RE. Ovarian teratoma with glial implants on the peritoneum: an analysis of 12 cases. Hum Pathol 1970;1:643-653.

5 Chuang J, Chen L. Ovarian teratoma with gliomatosis peritonei. J Pediatr Surg 1992;27:662-664.

6 Hill DA, Dehner LP, White FV et al. Gliomatosis peritonei as a complication of a ventriculoperitoneal shunt: case report and review of the literature. J Pediatr Surg 2000;35:497-499.

7 Lobotesis K, U-King-Im JM, Cross JJ et al. Gliomatosis peritonei associated with a ventriculo-peritoneal shunt. Clin Radiol 2009;64:95-99.

8 Lovell MA, Ross GW, Cooper PH. Gliomatosis peritonei associated with a ventriculoperitoneal shunt. Am J Clin Pathol 1989;91:485-487.

9 Kurman RJ, Carcangiu ML, Herrington CS et al. (eds). WHO Classification of Tumours of Female Reproductive Organs, 4th edn. IARC Press: Lyon, France, 2014, $101 \mathrm{pp}$.

10 Yoon NR, Lee JW, Kim BG et al. Gliomatosis peritonei is associated with frequent recurrence, but does not affect overall survival in patients with ovarian immature teratoma. Virchows Arch 2012;461:299-304.

11 Shefren G, Collin J, Soriero O. Gliomatosis peritonei with malignant transformation: a case report and review of the literature. Am J Obstet Gynecol 1991;164: 1617-1620.

12 Dadmanesh F, Miller DM, Swenerton KD et al. Gliomatosis peritonei with malignant transformation. Mod Pathol 1997;10:597-601.

13 O'Connor DM, Norris HJ. The influence of grade on the outcome of stage I ovarian immature (malignant) teratomas and the reproducibility of grading. Int J Gynecol Pathol 1994;13:283-289.

14 Prat J, FIGO Committee on Gynecologic Oncology. Staging classification for cancer of the ovary, fallopian tube, and peritoneum. Int J Gynaecol Obstet 2014;124: $1-5$.

15 Bentivegna E, Gonthier C, Uzan C et al. Gliomatosis peritonei: a particular entity with specific outcomes 
within the growing teratoma syndrome. Int J Gynecol Cancer 2015;25:244-249.

16 Daher P, Riachy E, Khoury A et al. Growing teratoma syndrome: first case report in a 4-year-old girl. J Pediatr Adolesc Gynecol 2015;28:e5-e7.

17 Djordjevic B, Euscher ED, Malpica A. Growing teratoma syndrome of the ovary: review of literature and first report of a carcinoid tumor arising in a growing teratoma of the ovary. Am J Surg Pathol 2007;31:1913-1918.

18 Kato N, Uchigasaki S, Fukase M. How does secondary neoplasm arise from mature teratomas in growing teratoma syndrome of the ovary? A report of two cases. Pathol Int 2013;63:607-610.

19 Logothetis CJ, Samuels ML, Trindade A et al. The growing teratoma syndrome. Cancer 1982;50:1629-1635.

20 Mrabti H, El Ghissassi I, Sbitti Y et al. Growing teratoma syndrome and peritoneal gliomatosis. Case Rep Med 2011;2011:123527.

21 Nogales FF, Preda O, Dulcey I. Gliomatosis peritonei as a natural experiment in tissue differentiation. Int J Dev Biol 2012:56:969-974.

22 Takahashi K, Tanabe K, Ohnuki M et al. Induction of pluripotent stem cells from adult human fibroblasts by defined factors. Cell 2007;131:861-872.
23 Takahashi K, Yamanaka S. Induction of pluripotent stem cells from mouse embryonic and adult fibroblast cultures by defined factors. Cell 2006;126:663-676.

24 Zhang S, Cui W. Sox2, a key factor in the regulation of pluripotency and neural differentiation. World J Stem Cells 2014;6:305-311.

25 Maucksch C, Jones KS, Connor B. Concise review: the involvement of SOX2 in direct reprogramming of induced neural stem/precursor cells. Stem Cells Transl Med 2013;2:579-583.

26 Phi JH, Park SH, Paek SH et al. Expression of Sox2 in mature and immature teratomas of central nervous system. Mod Pathol 2007;20:742-748.

27 Phi JH, Park SH, Kim SK et al. Sox2 expression in brain tumors: a reflection of the neuroglial differentiation pathway. Am J Surg Pathol 2008;32:103-112.

28 Nogales FF, Dulcey I, Preda O. Germ cell tumors of the ovary: an update. Arch Pathol Lab Med 2014;138:351-362.

29 Ferguson AW, Katabuchi H, Ronnett BM et al. Glial implants in gliomatosis peritonei arise from normal tissue, not from the associated teratoma. Am J Pathol 2001;159:51-55.

30 Kwan MY, Kalle W, Lau GT et al. Is gliomatosis peritonei derived from the associated ovarian teratoma? Hum Pathol 2004;35:685-688. 